\title{
SOME THEOREMS ABOUT THE RIESZ FRACTIONAL INTEGRAL
}

\author{
BY \\ NICOLAAS DU PLESSIS
}

I show in this paper that theorems which hold for Riemann-Liouville fractional integrals have analogues holding for the Riesz fractional integral [1]. Theorems 1, 2, and 3 are analogous to well-known results due to Hardy and Littlewood [2]. Theorem 4 is of a different character and is analogous to one recently proved by the author [3].

The Riesz fractional integral $f_{\alpha}(P)$ of order $\alpha$ is given by

$$
f_{\alpha}(P)=K_{m}^{-1} \int_{E} r_{P Q}^{\alpha-m} f(Q) d Q, \text { where } K_{m}=\pi^{m / 2} 2^{\alpha} \Gamma(\alpha / 2)[\Gamma((m-\alpha) / 2)]^{-1},
$$

$E$ denotes all of Euclidean $m$-space, and $r_{P Q}$ denotes the distance between $P$ and $Q$.

We assume always that $f(Q)$ is $L$-integrable over $E$.

I prove the following theorems.

Theorem 1. If $f(P) \in \operatorname{Lip} \beta, 0<\beta<1$ then $f_{\alpha}(P) \in \operatorname{Lip}(\alpha+\beta), 0<\alpha+\beta<1$.

Theorem 2. If $f(P) \in L^{q}, q>1,1+m / q>\alpha>m / q$, then

$$
f_{\alpha}(P) \in \operatorname{lip}(\alpha-\dot{m} / q) \text {. }
$$

Theorem 3. If $f(P) \in L^{q}$ and $0<\alpha<m / q$, then

$$
f_{\alpha}(P) \in L^{r}, \text { where } \alpha=m(1 / q-1 / r) .
$$

TheOREM 4. If $f(P) \in L^{q}$ then

(a) for $0<\alpha<m, 2<q<\infty, f_{\alpha / q}(P)$ is finite everywhere except possibly in a set which is of zero $\beta$-capacity for all $\beta>m-\alpha$;

(b) for $0<\alpha<m, 1 \leqq q \leqq 2, f_{\alpha / q}(P)$ is finite everywhere except possibly in a set of zero $(m-\alpha)$-capacity.

Both (a) and (b) are best possible.

1. Preliminaries. If $P$ is the point $\left(x_{1}, \cdots, x_{m}\right)$ and $Q$ the point $\left(t_{1}, \cdots, t_{m}\right)$ we define the points $\left(x_{1}+t_{1}, \cdots, x_{m}+t_{m}\right)$ and $\left(x_{1}-t_{1}, \cdots, x_{m}-t_{m}\right)$ to be $P+Q$ and $P-Q$ respectively. The distance $|P|$ of $P$ from the origin $0=\left(0_{1} \cdots, 0\right)$ is given by $|P|^{2}=\sum_{r-1}^{m} x_{r}^{2}$, and $|P-Q|$ is the distance $P$ to $Q$.

If, for $0 \leqq \beta \leqq 1, f(P+H)-f(P)=O\left(|H|^{\beta}\right)$ uniformly in $P$ as $|H| \rightarrow 0$, we say that $f(P) \in \operatorname{Lip} \beta$. If, in this, $O$ is replaced by $o$ we say that $f(P) \in \operatorname{lip} \beta$.

Received by the editors July 26, 1954. 
Next, we have

$$
\begin{aligned}
& K_{m}\left(f_{\alpha}(P+H)-f_{\alpha}(P)\right) \\
& =\left(\int_{U}+\int_{E-U}\right)\left(|Q-H|^{\alpha-m}-|Q|^{\alpha-m}\right) f(Q+P) d Q,
\end{aligned}
$$

where $U$ is the unit hypersphere having the origin as center. For $|H|<1 / 2$ it is not difficult to establish that

$$
|Q-H|^{\alpha-m}-|Q|^{\alpha-m}=O(|H|)
$$

uniformly in $E-U$. The second integral is thus $O(H)$ uniformly in $P$, and so

$$
\begin{aligned}
K_{m}\left(f_{\alpha}(P+H)-\right. & \left.f_{\alpha}(P)\right) \\
& =\int_{U}\left(|Q-H|^{\alpha-m}-|Q|^{\alpha-m}\right) f(Q+P) d Q+O(|H|) .
\end{aligned}
$$

2. Proofs of Theorems 1 and 2. First, Theorem 1. The first term on the right-hand side of (1) of $\$ 1$ may be rewritten in the form

$$
\begin{aligned}
\int_{U}\left(|Q-H|^{\alpha-m}-|Q|^{\alpha-m}\right)( & (Q+P)-f(P)) d Q \\
+ & f(P)\left\{\int_{U^{\prime}}|Q|^{\alpha-m} d Q-\int_{U}|Q|^{\alpha-m} d Q\right\},
\end{aligned}
$$

where $U^{\prime}$ is the sphere $U$ transforms into under the transformation $Q^{\prime}=Q-H$. The expression in curly brackets is dominated by $\int_{S}|Q|^{\alpha-m} d Q$, where $S=U^{\prime}+U-U^{\prime} U$.

Now $m S<\pi^{m / 2}[\Gamma((m+2) / 2)]^{-1}\left\{(1+|H|)^{m}-1\right\}=O(|H|)$ and $|Q|^{\alpha-m}$ $<2^{m-\alpha}$ in $S$ for $|H|<1 / 2$. Consequently, the second term in (2) is $O(|H|)$.

To deal with the first term we note that it is of order $\int_{U}|| Q-\left.H\right|^{\alpha-m}$ $-\left.|Q|^{a-m}|| Q\right|^{\beta} d Q$ and apply a uniform dilatation transformation of ratio 1 : $|H|$ and then a rotation which takes the transform of $H$ into the point $1=(1,0, \cdots, 0)$. The first term is then seen to be less than

$$
|H|^{\alpha+\beta} \int_{E}|| Q-\left.1\right|^{\alpha-m}-\left.|Q|^{\alpha-m}|| Q\right|^{\beta} d Q=O\left(|H|^{\alpha+\beta}\right),
$$

since it is again a simple matter to establish that the integral is finite. This proves Theorem 1.

Next, Theorem 2. Let $S(r)$ denote the hypersphere of radius $r$ centered at the origin and write

$$
A(\delta)=S(\delta)-S(|H|), \quad B(\delta)=U-S(\delta),
$$

where $\delta$ will presently be defined. Split the right-hand side of (1) into integrals 
$I_{1}$ over $S(|H|), I_{2}$ over $A(\delta)$, and $I_{3}$ over $B(\delta)$. Then, firstly

$$
\begin{aligned}
\left|I_{1}\right| \leqq & \left\{\int_{S(|H|)}|| Q-\left.H\right|^{\alpha-m}-\left.|Q|^{\alpha-m}\right|^{\prime} d Q\right\}^{1 / q^{\prime}} \\
& \cdot\left\{\int_{S(|H|)}|f(Q+P)|^{q} d Q\right\}^{1 / q} \\
= & |H|^{\alpha-m / q}\left\{\int_{U}|| Q-\left.1\right|^{\alpha-m}-|Q|^{\alpha-m} \mid q^{\prime} d Q\right\}^{1 / q^{\prime}} o(1)
\end{aligned}
$$

as $|H| \rightarrow 0$ : we use the same transformation on the integral as before. Thus $I=o\left(|H|^{\alpha-m / q}\right)$. Further

$$
\begin{aligned}
& \left|I_{2}\right| \leqq\left\{\int_{A(\delta)}|| Q-\left.H\right|^{\alpha-m}-|Q|^{\alpha-m} \mid q^{\prime} d Q\right\}^{1 / q^{\prime}} \\
& \left\{\int_{A(\delta)}|f(Q+P)|^{q} d Q\right\}^{1 / Q} \text {. }
\end{aligned}
$$

It is again easy to show that, for $|H|<\delta / 3$,

$$
|| Q-\left.H\right|^{\alpha-m}-\left.|Q|^{\alpha-m}|\leqq C| H|| Q\right|^{\alpha-m-1}
$$

and thus

$$
\left|I_{2}\right| \leqq C|H|\left\{\int_{A(\delta)}|Q|^{(\alpha-m-1) q^{\prime}} d Q\right\}^{1 / q^{\prime}}\left\{\int_{A(\delta)}|f(Q+P)|{ }^{q} d Q\right\}^{1 / q} .
$$

Further

$$
\int_{A(\delta)}|Q|^{(\alpha-m-1) q^{\prime}} d Q<|H|^{(\alpha-1) q^{\prime}-m\left(q^{\prime}-1\right)} \int_{E-U}|Q|^{(\alpha-m-1) q^{\prime}} d Q,
$$

so that

$$
\left|I_{2}\right| \leqq C|H|^{\alpha-m / q}\left\{\int_{A(\delta)}|f(Q+P)|^{a} d Q\right\}^{1 / q} .
$$

Given any $\epsilon>0$, we can choose $\delta$ so that $\int_{A(\delta)}|f(Q+P)|^{a} d Q$ is less than $(\epsilon / C)^{q}$, and so

Finally

$$
\left|I_{2}\right|<\epsilon|H|^{\alpha-m / q} \text {. }
$$

$$
\left|I_{3}\right| \leqq\left\{\int_{B(\delta)}|| Q-\left.H\right|^{\alpha-m}-|Q|^{\alpha-m} \mid q^{\prime} d Q\right\}^{1 / q^{\prime}}\left\{\int_{E}|f(Q+P)|^{q} d Q\right\}^{1 / q} .
$$

For fixed $\delta,|Q-H|^{\alpha-m}-|Q|^{\alpha-m}=O(|H|)$ uniformly in $B(\delta)$, and so $I_{8}=O(|H|)$. 
Thus, finally, $K_{m}\left(f_{\alpha}(P+H)-f_{\alpha}(P)\right)=o\left(|H|^{\alpha-m / q}\right)$, giving the required result.

3. Proof of Theorem 3. We first prove a many-dimensional generalization of a theorem due to Hardy and Littlewood [2, Theorem 3].

Lemma. If $f(P) \in L^{q}, g(Q) \in L^{r}, 1 / q+1 / r>1, q>1, r>1$ and $\mu=2-1 / q$ $-1 / r$ then

$$
\int_{E} \int_{E}|Q-P|-m \mu f(P) g(Q) d P d Q \leqq K M_{Q}(f) M_{r}(g),
$$

where $M_{Q}(f)=\left\{\int_{E}|f(P)|^{a} d P\right\}^{1 / q}$ and $M_{r}(g)$ is similarly defined.

I prove here the case $m=3$, which is sufficiently typical.

Since an arithmetic mean is greater than the corresponding geometric mean we have

$$
\begin{aligned}
|P-Q|^{2} & =\left(x_{1}-t_{1}\right)^{2}+\left(x_{2}-t_{2}\right)^{2}+\left(x_{3}-t_{3}\right)^{2} \\
& \geqq 3\left|x_{1}-t_{1}\right|^{2 / 3}\left|x_{2}-t_{2}\right|^{2 / 3}\left|x_{3}-t_{3}\right|^{2 / 3}
\end{aligned}
$$

and so

$$
|P-Q|^{-3 \mu} \leqq C\left|x_{1}-t_{1}\right|^{-\mu}\left|x_{2}-t_{2}\right|^{-\mu}\left|x_{3}-t_{3}\right|^{-\mu} .
$$

Consequently the left-hand side of (1) is not greater than a constant multiple of

$$
\begin{array}{r}
\int_{-\infty}^{\infty} \int_{-\infty}^{\infty} \int_{-\infty}^{\infty} \int_{-\infty}^{\infty} \int_{-\infty}^{\infty} \int_{-\infty}^{\infty} \frac{f\left(x_{1}, x_{2}, x_{3}\right) g\left(t_{1}, t_{2}, t_{3}\right)}{\left|x_{1}-t_{1}\right|^{\mu}\left|x_{2}-t_{2}\right|^{\mu}\left|x_{3}-t_{3}\right|^{\mu}} \\
\cdot d t_{3} d x_{3} d t_{2} d x_{2} d t_{1} d x_{1} .
\end{array}
$$

By the Hardy-Littlewood theorem mentioned, which is the case $m=1$ of the lemma,

$$
\left.\int_{-\infty}^{\infty} \int_{-\infty}^{\infty}\left|x_{3}-t_{3}\right|\right|^{-\mu} f\left(x_{1}, x_{2}, x_{3}\right) g\left(t_{1}, t_{2}, t_{3}\right) d t_{3} d x_{3}
$$

is dominated by $C F\left(x_{1}, x_{2}\right) G\left(t_{1}, t_{2}\right)$, where $F\left(x_{1}, x_{2}\right)=\left\{\int_{-\infty}^{\infty}\left|f\left(x_{1}, x_{2}, x_{3}\right)\right|^{a} d x_{3}\right\}^{1 / 4}$ and $G\left(t_{1}, t_{2}\right)$ is defined analogously.

Hence [2] is dominated by

$$
C_{1} \int_{-\infty}^{\infty} \int_{-\infty}^{\infty} \int_{-\infty}^{\infty} \int_{-\infty}^{\infty}\left|x_{1}-t_{1}\right|^{-\mu}\left|x_{2}-t_{2}\right|^{-\mu} F\left(x_{1}, x_{2}\right) G\left(t_{1}, t_{2}\right) d t_{2} d x_{2} d t_{1} d x_{1} .
$$

Applying the case $m=1$ of the lemma again to the inner two integrals we find that (1) is dominated by 


$$
C_{1} C_{2} \int_{-\infty}^{\infty} \int_{-\infty}^{\infty}\left|x_{1}-t_{1}\right|-\mu F\left(x_{1}\right) G\left(t_{1}\right) d t_{1} d x_{1},
$$

where $F\left(x_{1}\right)=\left\{\int_{-\infty}^{\infty}\left|F\left(x_{1}, x_{2}\right)\right|^{q} d x_{2}\right\}^{1 / q}$ and $G\left(t_{1}\right)$ is defined analogously.

A final application of the lemma with $m=1$ shows that (1) is dominated by $C_{1} C_{2} C_{3} M_{q}(F) M_{r}(G)$. Since $M_{q}(F)$ equals $\left\{\int_{E}|f(P)|^{q} d P\right\}^{1 / q}$ and a similar result holds for $M_{r}(G)$, we have the required result.

To prove Theorem 3 it is sufficient to prove that, for every $g(P)$ such that $M_{r^{\prime}}(g) \leqq 1$,

$$
\int_{E} f_{\alpha}(P) g(P) d P \leqq K M_{q}(f) .
$$

The left-hand side of this is equal to

$$
K_{m}^{-1} \int_{E} \int_{E}|P-Q|^{\alpha-m} f(Q) g(P) d Q d P
$$

and, since $\alpha-m=m(1 / q-1 / r)-m=-m\left(2-1 / q-1 / r^{\prime}\right)$, the lemma applies and shows (3) to be, in modulus, not greater than $K^{\prime} M_{q}(f) M_{r^{\prime}}(g) \leqq K^{\prime} M_{q}(f)$, thus proving the theorem.

4. Preliminaries about Theorem 4. We say, with Frostman $[4$, p. 26], that a non-negative additive set function $\mu(S)$ defined for all Borel sets in $E$ is a distribution if $\mu(E)=1$. Further, if $S \subset E$ and $\mu(S)=1$ we say that the distribution is concentrated on $S$.

Let $S$ be a given set. Suppose that there is a distribution concentrated on $S$ such that

$$
V_{\beta}=\sup _{P \in E} \int_{E}|Q-P|^{-\beta} d \mu(Q)
$$

is finite. Then we say that $S$ is of positive $\beta$-capacity. Otherwise $S$ is said to be of zero $\beta$-capacity. Clearly, if $S$ is of positive $\beta$-capacity, it is of positive $\gamma$-capacity for all $\gamma<\beta$. Further, if it is of zero $\beta$-capacity, it is of zero $\gamma$ capacity for all $\gamma>\beta$.

Lemma. For $1<q<2$, and for every $\epsilon>0$ for which $q-\epsilon>1$, we have

$$
\int_{S}\left\{\int_{E}|Q-P|^{\left(\alpha / q^{\prime}\right)-m} d \mu(Q)\right\}^{q-\epsilon} d P \leqq A(\alpha, \epsilon, m, q, S) V_{m-\alpha}^{(\alpha-\epsilon) /(q-\epsilon)^{\prime}},
$$

where $A(\alpha, \epsilon, m, q, S)$ is a constant depending only on the parameters shown and $S$ is a bounded set.

For $2 \leqq q \leqq \infty$ we have

$$
\int_{E}\left\{\int_{E}|Q-P|^{\left(\alpha / \alpha^{\prime}\right)-m} d \mu(Q)\right\}^{q} d P \leqq A(\alpha, m) V_{m-\alpha}^{q-1}
$$


where $A(\alpha, m)$ is a constant depending only on the parameters shown.

We have

$$
\begin{aligned}
& \left\{\int_{E}|Q-P|^{\left(\alpha / q^{\prime}\right)-m} d \mu(Q)\right\}^{q-\epsilon}=\left\{\int_{E}|Q-P|^{-\alpha / q}|Q-P|^{\alpha-m} d \mu(Q)\right\}^{q-\epsilon} \\
& \leqq\left\{\int_{E}|Q-P|-\alpha(q-\epsilon) / q|Q-P|^{\alpha-m} d \mu(Q)\right\} \\
& \cdot\left\{\int_{E}|Q-P| \alpha-m d \mu(Q)\right\}^{(q-\epsilon) /(q-\epsilon)^{\prime}}
\end{aligned}
$$

by Hölder's inequality. The second factor is not greater than $V^{(q-e) /(q-e) \prime}$ while the first is $\int_{E}|Q-P|^{\alpha_{e} / q-m} d \mu(Q)$. The left-hand side of (1) is therefore not greater than

$$
V_{m-\alpha}^{(q-\epsilon) /(q-\epsilon)^{\prime}} \int_{S} d P \int_{E}|Q-P|^{\alpha \epsilon / q-m} d \mu(Q) .
$$

We invert the order of integration and note that

$$
\int_{S}|Q-P|^{\alpha \epsilon / q-m} d P=A(\alpha, \epsilon, m, q, S), \text { say. }
$$

Furthermore $\int_{E} d \mu(Q)=1$. (1) now follows.

To prove (2) I first show the result true for $q=2$ and then that this implies its truth for $q>2$. For this latter part of the proof I am indebted to Professor J. E. Littlewood.

We have first, on inverting the order of integration,

$$
\begin{aligned}
\int_{E}\left\{\int_{E} \mid Q-\right. & \left.\left.P\right|^{(\alpha-m) / 2} d \mu(Q)\right\}^{2} d P \\
& =\int_{E} \int_{E} \int_{E}|Q-P|(\alpha-m) / 2|R-P|(\alpha-m) / 2 d P d \mu(Q) d \mu(R) .
\end{aligned}
$$

To deal with the inner integral we dilate $E$ uniformly, taking $Q$ as the center of dilatation, in the ratio $1:|Q-R|$ and then rotate the dilated space so that the transform of $Q-R$ goes into the point 1 . The inner integral then becomes

$$
|Q-R|^{\alpha-m} \int_{E}|U|^{(\alpha-m) / 2}|U+1|^{(\alpha-m) / 2} d U=B(\alpha, m)|Q-R|^{\alpha-m} .
$$

Consequently, the right-hand side of (3) is dominated by

$$
B(\alpha, m) \int_{E} \int_{E}|Q-R|^{\alpha-m} d \mu(Q) \leqq B(\alpha, m) V_{m-\alpha} \mu(E) .
$$


Since $\mu(E)=1$ this gives the result for $q=2$.

For $q>2$, we have

$$
\begin{aligned}
& \int_{E}\left\{\int_{E}|Q-P|^{\alpha / q^{\prime}-m} d \mu(Q)\right\}^{q} d P \\
& =\int_{E}\left\{\int_{E}|Q-P|^{((q-2) / q)(\alpha-m)}|Q-P|^{(\alpha-2 m) / q} d \mu(Q)\right\}^{q} d P
\end{aligned}
$$

and this, by Hölder's inequality, does not exceed

$$
J=\int_{E}\left\{\int_{E}|Q-P|^{\alpha-m} d \mu(Q)\right\}^{q-2}\left\{\int_{E}|Q-P|^{(\alpha-m) / 2} d \mu(Q)\right\}^{2} d P .
$$

The first curly bracket does not exceed $V_{m-\alpha}^{q-2}$ (by the definition of $V_{m-\alpha}$ ). So

$$
J \leqq V_{m-\alpha}^{q-2} \int_{E}\left\{\int_{E}|Q-P|^{(\alpha-m) / 2} d \mu(Q)\right\}^{2} d P
$$

and this, by the result for $q=2$, does not exceed $V_{m-\alpha}^{q-2} B(\alpha, m) V_{m-\alpha}$. This gives the result for $q>2$.

5. Proof of Theorem 4. Let

$$
S_{n}(P)=\int_{E}|Q-P|^{\alpha / q-m}[f(Q)]_{n} d Q,
$$

where

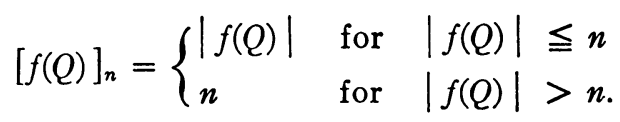

$S_{n}(P)$ is always defined and finite, and to prove the theorem it is sufficient to show that $S_{n}(P)$ is bounded everywhere except possibly in a set of zero $\beta$-capacity, where $\beta=m-\alpha$ for $1 \leqq q \leqq 2$ and $\beta>m-\alpha$ for $q>2$.

Assume, then, that $S_{n}(P)$ is unbounded in a set $M$ of positive $\beta$-capacity. It is then unbounded in a bounded set $S$ of positive $\beta$-capacity. Then, first, there is a distribution concentrated on $S$ such that $\int_{E}|Q-P|^{-\beta} d \mu(Q)$ is bounded for all $P$. Secondly, there is a function $n(P) \leqq n$, taking only integer values such that $\int_{S} S_{n(P)}(P) d \mu(P)$ exists and is unbounded as $n \rightarrow \infty$. This is an adaptation of a known result used by Salem and Zygmund [5, embodied in the proof of Theorem II], but a proof is perhaps not unwelcome.

Let $\bar{S}_{n}(P)=\sup S_{m}(P)$ for $0 \leqq m \leqq n$. Then for all $P \in S,\left\{\bar{S}_{n}(P)\right\}^{-1} \rightarrow 0$ as $n \rightarrow \infty$. By Egoroff's theorem on uniform convergence it follows that there is a set $S^{\prime} \subset S$ such that $\mu\left(S-S^{\prime}\right)$ is as small as we please, and in which $\left\{\bar{S}_{n}(P)\right\}^{-1} \rightarrow 0$ uniformly. It follows that, given any large number $G$, there is an integer $n_{0}=n_{0}(G)$ such that, for all $P \in S^{\prime}, \bar{S}_{n}(P)>G$ for all $n>n_{0}(G)$ Choose $n(P)$ such that $S_{n(P)}(P)=\bar{S}_{n}(P)$. Then 


$$
\int_{S} S_{n(P)}(P) d \mu(P)>G \mu\left(S^{\prime}\right) \text { for } n>n_{0}
$$

and so

$$
\int_{S} S_{n(P)}(P) d \mu(P) \rightarrow+\infty \quad \text { as } n \rightarrow \infty \text {. }
$$

I show this last to be impossible. We have

$$
\begin{aligned}
\left|\int_{S} S_{n(P)}(P) d \mu(P)\right| & =\left|\int_{S} \int_{E}\right| Q-\left.P\right|^{\alpha / q-m}[f(Q)]_{n(P)} d Q d \mu(P) \mid \\
& \leqq \int_{E}|f(Q)| \int_{S}|Q-P|^{\alpha / q-m} d \mu(P) d Q
\end{aligned}
$$

and this does not exceed $M_{q}(f) M_{q^{\prime}}\left[\int_{S}|Q-P|^{\alpha / q-m} d \mu(P)\right]$. Now $M_{q}(f)<+\infty$ by hypothesis, and we have only to show that

$$
M_{q^{\prime}}\left[\int_{E}|Q-P|^{\alpha / q-m} d \mu(P)\right]
$$

is bounded.

If $1 \leqq q \leqq 2$ then $q^{\prime} \geqq 2$ and (2) of the lemma of $\$ 4$ immediately gives (1). If $q>2$ we write $\beta=m-\gamma$. Since $\gamma<\alpha$ there is an $r<q$ such that $\alpha / q=\gamma / r$. We may suppose $\beta$ so near $m-\alpha$ that $2<r<q$ since the result, if true for a given $\beta$, is true for a larger $\beta$. We may now rewrite (1) in the form

$$
M_{r^{\prime}-\epsilon}\left[\int_{E}|P-Q| \gamma / r-m d \mu(Q)\right]
$$

which, since $r^{\prime}>2$, is shown to be bounded by invoking (1) of the lemma.

6. Theorem 4 is best possible. We show this by constructing a function $f(P) \in L^{q}$ and a set $M$ of positive $\beta$-capacity (where $\beta=m-\alpha$ when $1 \leqq q \leqq 2$, and $\beta$ is any number greater than $m-\alpha$ when $q>2)$ at every point of which $f_{\alpha / q}(P)$ is infinite. It will avoid unnecessary complication and fully illustrate the general procedure if this is done for the simplest case $m=2$.

$M$ is constructed as follows. Let $\left\{\xi_{n}\right\}$ be any sequence such that $0<\xi_{n}$ $<1 / 2$. Let $M_{0}$ be the unit square $0 \leqq x_{1} \leqq 1,0 \leqq x_{2} \leqq 1$. From $M_{0}$ remove the rectangle $\xi_{1}<x_{1}<1-\xi_{1}, 0 \leqq x_{2} \leqq 1$ thus leaving the set $M_{1}$. From the lefthand rectangle in $M_{1}$ remove the rectangle $\xi_{1} \xi_{2}<x_{1}<\xi_{1}\left(1-\xi_{2}\right), 0 \leqq x_{2} \leqq 1$, and make a similar symmetric removal from the right-hand rectangle of $M_{1}$, thus leaving a set consisting of 4 closed rectangles of length 1 and breadth $\xi_{1} \xi_{2}$. If we continue in this manner we are left, after the $n$th removal, with a set $M_{n}$ consisting of $2^{n}$ closed rectangles each of length 1 and breadth $\xi_{1} \xi_{2}$ ... $\xi_{n}$. Consequently 


$$
m M_{n}=2^{n} \xi_{1} \xi_{2} \cdots \xi_{n} .
$$

It is known $[5$, p. 40$]$ that the projection $S$ of $M=\lim M_{n}$ on the $x$-axis will be of positive $\beta$-capacity if and only if

$$
\sum_{n=1}^{\infty} 2^{-n}\left(\xi_{1} \xi_{2} \cdots \xi_{n}\right)^{-\beta}<\infty
$$

If $S$ is of positive $\beta$-capacity there is a distribution $\nu$ concentrated on $S$ such that $\int_{0}^{1}\left|x_{1}-t\right|^{-\beta} d \nu(t)$ is bounded for all $x_{1}$. Let $\mu$ be an additive set function defined over $E$ by

$$
\mu(X)=\iint_{X} d \nu\left(x_{1}\right) d x_{2}
$$

Then

$$
\int_{M}|P-Q|^{-\beta-1} d \mu(Q)=\int_{0}^{1} \int_{0}^{1}\left[\left(x_{1}-t_{1}\right)^{2}+\left(x_{2}-t_{2}\right)^{2}\right]^{-(\beta+1) / 2} d t_{2} d \nu\left(t_{1}\right) .
$$

In the inner integral make the substitution $x_{2}-t_{2}=\left(x_{1}-t_{1}\right) u$. It is then dominated by

$$
\left|x_{1}-t_{1}\right|^{-\beta} \int_{-\infty}^{\infty}\left(1+u^{2}\right)^{-(\beta+1) / 2} d u=A(\beta)\left|x_{1}-t_{1}\right|^{-\beta} .
$$

Consequently, since $\mu$ is a distribution concentrated on $M$,

$$
\int_{M}|P-Q|^{-\beta-1} d \mu(Q)=\int_{E}|P-Q|^{-\beta-1} d \mu(Q) \leqq A(\beta) \int_{0}^{1}\left|x_{1}-t_{1}\right|^{-\beta} d \nu\left(t_{1}\right),
$$

which is bounded. Thus $M$ is of positive $(\beta+1)$-capacity if $S$ is of positive $\beta$-capacity.

Define $\left\{f_{n}(P)\right\}$ over $M_{0}$ by

$$
\begin{aligned}
& f_{0}(P)=0 \text { in } M_{0}, \\
& f_{n}(P)=\left(\xi_{1} \xi_{2} \cdots \xi_{n}\right)^{-\alpha / q_{n}} n^{-1} \text { in } M_{n}, \\
& f_{n}(P)=f_{n-1}(P) \text { in } M_{0}-M_{n} .
\end{aligned}
$$

Since $\left\{f_{n}(P)\right\}$ is, eventually, an increasing sequence of measurable functions the function $f(P)$ given by

$$
\begin{aligned}
& f(P)=\lim _{n \rightarrow \infty} f_{n}(P) \text { in } M_{0}, \\
& f(P)=0 \text { in } E-M_{0}
\end{aligned}
$$

exists and is measurable over $E$.

It is easily seen that, for $n=1,2, \cdots$, 


$$
\begin{aligned}
& f(P)=0 \text { in } M_{0}-M_{1}, \\
& f(P)=\left(\xi_{1} \xi_{2} \cdots \xi_{n}\right)^{-\alpha / q_{n}-1} \text { on } M_{n}-M_{n+1}
\end{aligned}
$$

so that

$$
\begin{aligned}
\int_{E}|f(P)|^{q} d P & =\int_{M_{0}}|f(P)|^{q} d P=\sum_{n=1}^{\infty} \int_{M_{n}-M_{n+1}}|f(P)|^{q} d P \\
& =\sum_{n=1}^{\infty}\left(\xi_{1} \xi_{2} \cdots \xi_{n}\right)^{-\alpha} n^{-q}\left(m M_{n}-m M_{n+1}\right) \\
& =\sum_{n=1}^{\infty}\left(1-2 \xi_{n+1}\right) 2^{n}\left(\xi_{1} \xi_{2} \cdots \xi_{n}\right)^{1-\alpha} n^{-q} .
\end{aligned}
$$

For $q>2$, we may choose $\delta>0$ so that $2(1+\delta)<q$, and then put

$$
2 \xi_{n}^{1-\alpha}=1+(1+\delta) n^{-1} .
$$

Then $2^{-n}\left(\xi_{1} \xi_{2} \cdots \xi_{n}\right)^{\alpha-1} \sim C n^{-1-\delta}$ so that (1) with $\beta=1-\alpha$ is satisfied, showing $S$ to be of positive $(1-\alpha)$-capacity, and hence that $M$ is of positive $(2-\alpha)$-capacity.

Further, (2) is clearly finite, so that $f \in L^{a}$ over $E$.

Let $P\left(x_{1}, x_{2}\right)$ be any point of $M$. Let

$$
\begin{array}{r}
M_{n}(P)=M_{n} \cdot S\left[t_{2} ; x_{2}-\epsilon_{n} \leqq t_{2} \leqq x_{2}+\epsilon_{n}\right], \quad \text { where } \epsilon_{n}=\xi_{1} \xi_{2} \cdots \xi_{n} / 2 ; \\
M_{n}^{*}(P)=\left(M_{n}-M_{n+1}\right) S\left[t_{2} ; x_{2}-\delta_{n} \leqq\right. \\
\left.\quad t_{2} \leqq x_{2}+\delta_{n}\right], \\
\quad \text { where } \delta_{n}=\xi_{1} \xi_{2} \cdots \xi_{n}\left(1-2 \xi_{n+1}\right) / 2 .
\end{array}
$$

$M_{n}(P)$ then consists of $2^{n}$ squares each of side $\xi_{1} \xi_{2} \cdots \xi_{n}$, while $M_{n}^{*}(P) \subset M_{n}(P)$ and consists of $2^{n}$ squares each of side $\xi_{1} \xi_{2} \cdots \xi_{n}\left(1-2 \xi_{n+1}\right)$. No square in $M_{n}^{*}(P)$ contains $P$, but one of the squares, $I_{n}$ (say), is contained in that one of the squares, $J_{n}$ (say), of $M_{n}(P)$ which itself contains $P$. Furthermore, the $I_{n}(n=1,2, \cdots)$ are disjoint.

Now $|Q-P|<2^{1 / 2} \xi_{1} \cdots \xi_{n}$ for $Q$ in $J_{n}$, and so certainly for $Q$ in $I_{n}$, and thus

$$
K_{2} f_{\alpha / q}(P)=\int_{M_{0}}|Q-P|^{\alpha / q-2} f(Q) d Q=\sum_{n=1}^{\infty} \int_{M_{n}-M_{n+1}} \geqq \sum_{n=1}^{\infty} \int_{I_{n}} .
$$

This last is not less than

$$
\begin{aligned}
\sum_{n=1}^{\infty}\left(2^{1 / 2} \xi_{1} \cdots \xi_{n}\right)^{\alpha / q-2}\left(\xi_{1} \cdots \xi_{n}\right)^{-\alpha / q} n^{-1}\left(\xi_{1} \cdots \xi_{n}\right)^{2}\left(1-2 \xi_{n+1}\right)^{2} \\
=2^{\alpha / 2 q-1} \sum_{n=1}^{\infty}\left(1-2 \xi_{n+1}\right)^{2} n^{-1}=+\infty .
\end{aligned}
$$


Consequently, $f_{\alpha / q}(P)$ is infinite at every point of $M$, giving the required example in the case of $q>2$, thus showing part (a) of Theorem 4 best possible.

For the case $q \leqq 2$, let $\beta$ be any positive number less than $1-\alpha$ and let $\xi$ be such that $2 \xi^{(1-\alpha+\beta) / 2}=1$. Consider the set $M$ with $\xi_{n}=\xi$ for all $n$. Since $2 \xi^{\beta}>1, M$ is of positive $(\beta+1)$-capacity. Defining $f(P)$ as before, we use exactly the same argument to show that $f_{\alpha / q}(P)=+\infty$ at every point of $M$. Furthermore, since $2 \xi^{1-\alpha}<1,(2)$ is bounded, so that $f \in L^{q}$.

This shows part (b) of Theorem 4 best possible.

7. The lemma of $\$ 4$ is best possible. Consider, e.g., (2) of the lemma. Suppose this is not the case, i.e. that there is an $\epsilon>0$ for which, in general,

$$
M_{q+\epsilon}\left[\int_{E}|Q-P| \alpha / q^{\prime}-m d \mu(Q)\right]<\infty .
$$

If, then, $f(P) \in L^{(a+e) '}$ we may say that

$$
\left|\int_{E} S_{n(P)}(P) d \mu(P)\right| \leqq M_{(q+\epsilon)^{\prime}}(f) M_{q+\epsilon}\left[\int_{E}|Q-P|^{\alpha / q^{\prime}-m} d \mu(Q)\right]
$$

which is bounded. This would imply that (b) of Theorem 4 is not best possible. Since it is best possible we have shown (2) best possible. A similar argument using (a) would show (1) best possible.

\section{REFERENCES}

1. M. Riesz, L'intégrale de Riemann-Liouville et le problème de Cauchy, Acta Math. vol. 81 (1950) pp. 1-222.

2. G. H. Hardy and J. E. Littlewood, Some properties of fractional integrals, Math. Zeit. vol. 27 (1928) pp. 565-606.

3. N. du Plessis, $A$ theorem about fractional integrals, Proc. Amer. Math. Soc. vol. 3 (1952) pp. $892-898$.

4. O. Frostman, Potentiel d'equilibre et capacité des ensembles, Meddel. fran Lunds Univ. Mat. Sem. vol. 3 (1935) pp. 1-118.

5. R. Salem and A. Zygmund, Capacity of sets and Fourier series, Trans. Amer. Math. Soc. vol. 59 (1946) pp. 23-41.

RHODES UNIVERSITY, Grahamstown, South Africa 